\title{
Low-Rank and Spectral-Spatial Sparse Unmixing for Hyperspectral Remote Sensing Imagery
}

\author{
Fan Li $(\mathbb{D}$ \\ Jiangxi Province Key Laboratory of Water Information Cooperative Sensing and Intelligent Processing, School of \\ Information Engineering, Nanchang Institute of Technology, Nanchang 330099, China \\ Correspondence should be addressed to Fan Li; lifan@nit.edu.cn
}

Received 19 September 2021; Accepted 8 October 2021; Published 31 October 2021

Academic Editor: Chi-Hua Chen

Copyright (c) 2021 Fan Li. This is an open access article distributed under the Creative Commons Attribution License, which permits unrestricted use, distribution, and reproduction in any medium, provided the original work is properly cited.

\begin{abstract}
Sparse unmixing is an important technique for hyperspectral data analysis. Most sparse unmixing algorithms underutilize the spatial and spectral information of the hyperspectral image, which is unfavourable for the accuracy of endmember identification and abundance estimation. We propose a new spectral unmixing method based on the low-rank representation model and spatial-weighted collaborative sparsity, aiming to exploit structural information in both the spatial and spectral domains for unmixing. The spatial weights are incorporated into the collaborative sparse regularization term to enhance the spatial continuity of the image. Meanwhile, the global low-rank constraint is employed to maintain the spatial low-dimensional structure of the image. The model is solved by the well-known alternating direction method of multiplier, in which the abundance coefficients and the spatial weights are updated iteratively in the inner and outer loops, respectively. Experimental results obtained from simulation and real data reveal the superior performance of the proposed algorithm on spectral unmixing.
\end{abstract}

\section{Introduction}

Hyperspectral remote sensing is considered to be a major breakthrough in remote sensing technology due to its high spectral resolutions and simultaneous acquisition of both images and spectra of objects $[1,2]$. Hyperspectral remote sensing images provide rich earth observation information, which shows great application potential in mineral resource exploration, precision agriculture, and environmental monitoring [3]. However, the insufficient spatial resolution of hyperspectral images leads to the emergence of a large number of mixed pixels, which influences the fine interpretation of hyperspectral images [4]. Spectral unmixing is an effective way to deal with mixed pixels. It obtains pure spectral signals of ground objects (endmembers) in the imaging area and their corresponding proportions (abundance) [5].

The observed spectrum of the mixed pixel is a mixture of endmember spectra, and unmixing is essentially an optimization problem for a given spectral mixture model. The linear mixture model (LMM) assumes that each pixel is formed by linearly combining spectral signatures of endmembers [3]. Many hyperspectral unmixing algorithms based on LMM have been proposed, among which sparse unmixing methodology has received extensive attention [6, 7]. Sparse unmixing is a semisupervised spectral unmixing method, which takes the spectral library as a priori and assumes that the spectra of the endmembers participating in the hyperspectral image can be found in the library [5]. Generally, the number of endmembers in each mixed pixel is very small relative to the number of spectral signatures in the library; that is, the corresponding abundance is sparse. Therefore, the sparse regression algorithm is adopted to select the optimal endmember subset from the spectral library and simultaneously estimate the fractional abundances [8].

Sparse unmixing can be regarded as solving the combinatorial optimization problem by a constrained sparse linear regression algorithm, in which sparsity-inducing term promotes the sparsity of the abundance solutions. For example, the sparse unmixing via variable splitting and augmented Lagrangian (SUnSAL) uses the $\ell_{1}$ norm to characterize the 
sparsity of each abundance vector [5]. Furthermore, the collaborative SUnSAL (CLSUnSAL) uses the $\ell_{2,1}$ norm to enforce the joint sparsity among all pixels [9].

The iterative reweighted $\ell_{1}$ norm minimization is confirmed to enhance the sparsity of solutions $[10,11]$. In the work of [12], a weighting factor is introduced in the sparse regularization term to reduce the solution space. The double reweighted sparse unmixing algorithm (DRSU) employs the double weighted $\ell_{1}$ norm to simultaneously reduce the nonzero rows in the abundance matrix corresponding to the actual endmembers and the nonzero values in each abundance vector [13].

Hyperspectral data presents a three-dimensional cube structure with 2-dimensional spatial information and 1dimensional spectral information of the target area [14, 15]. Taking spatial information as a priori knowledge greatly improves the unmixing performance, so it has become one of the research hotspots in the field of hyperspectral unmixing [16-18]. For instance, the total variation (TV) regularizer is proposed to integrate the spatial-context information $[19,20]$, and the TV regularization term is added to the SUnSAL algorithm to construct the SUnSAL-TV algorithm [19]. Moreover, the local collaborative sparse unmixing algorithm (LCSU) focuses on the local collaborative characteristics of the image, which imposes different spatial weights on each local region of the abundance map [21].

Low rankness is another inherent characteristic of the abundance matrix, which provides a new perspective for exploring the spatial structure of the data $[22,23]$. The high spatial correlation of pixels in the image reflects in the high spectral similarity of these pixels. The similar pixels usually share the same endmembers and similar abundance maps, which means that the corresponding abundance vectors are linearly dependent; that is, the abundance matrix is low rank or approximately low rank. The low-rank constraint helps to obtain a low-rank approximation of the abundance matrix, which captures the global spatial data structure. The alternating direction sparse and low-rank unmixing (ADSpLRU) algorithm first attempts to combine sparse and low-rank constraints, which considers the spatial correlation of the pixels in a $3 \times 3$ sliding window [24]. The joint-sparseblocks and low-rank unmixing (JSpBLRU) algorithm divides the abundance matrix into flexible regular blocks with one row and multiple columns and applies joint sparsity to the blocks, and the low-rank regularizer is merged into the block sparse model synchronously [25].

In this paper, a low-rank and spectral-spatial sparse unmixing algorithm is proposed, which provides the low-rank constraint and the collaborative sparse constraint incorporating neighborhood spatial weights. The multiconstraint strategy effectively exploits the spatial and spectral information of the image and improves the accuracy of the abundance estimation.

The rest of this paper is structured as follows. Section 2 introduces the sparse unmixing model and its variants. Section 3 presents the low-rank and spectral-spatial sparse unmixing model and its optimization algorithm in detail. In Section 4, the effectiveness of the proposed algorithm is demonstrated by experiments on simulated and real hyperspectral data. Section 5 draws conclusions and suggests possible future research.

\section{Related Works}

Sparse unmixing replaces the endmember set used in LMM with a large spectral library and searches the library for the combination of spectral signatures that best represents the hyperspectral images [5]. Let $\mathbf{Y} \in \mathbb{R}^{l \times n}$ be a hyperspectral image including $n$ pixels with $l$ bands. The sparse unmixing model can be expressed as

$$
\mathbf{Y}=\mathbf{A X}+\mathbf{N}
$$

where $\mathbf{A} \in \mathbb{R}^{l \times m}$ is a spectral library including $m$ spectral signatures, $\mathbf{X} \in \mathbb{R}^{m \times n}$ is the abundance matrix corresponding to the spectral library $\mathbf{A}$, and $\mathbf{N} \in \mathbb{R}^{l \times n}$ is the noise or model error. The abundance matrix $\mathbf{X}$ reflects the proportion of spectral signatures from the library $\mathbf{A}$ in each pixel. According to the physical meaning of abundance, two constraints are considered: abundance nonnegativity constraint (ANC) and abundance sum-to-one constraint (ASC), which can be written as $\mathbf{X} \geq 0$ and $\mathbf{1}^{T} \mathbf{X}=\mathbf{1}$.

In fact, there are only a few entries from the spectral library $\mathbf{A}$ act on the mixed pixels, which results in many zeros in the relevant fractional abundances. That is, the abundance matrix $\mathbf{X}$ is sparse. Based on sparse and redundant representation theory, the unmixing problem can be modeled as follows:

$$
\begin{gathered}
\min _{\mathbf{X}} \frac{1}{2}\|\mathbf{Y}-\mathbf{A} \mathbf{X}\|_{F}^{2}+\lambda\|\mathbf{X}\|_{0} \\
\text { s.t. } \quad \mathbf{X} \geq 0
\end{gathered}
$$

where $\|\cdot\|_{F}$ is the Frobenius norm, $\lambda$ is a nonnegative regularization parameter, and $\|\mathbf{X}\|_{0}$ indicates the number of nonzero values in $\mathbf{X}$. Note that the ASC is not strictly conducted in the model (2), which is ascribed to the drawbacks mentioned in [5].

The optimization problem related to model (2) is NPhard. Under the condition of restricted isometric property (RIP), $\ell_{0}$ norm can be relaxed to $\ell_{1}$ norm [26]. The model (2) is modified as

$$
\begin{gathered}
\min _{\mathbf{X}} \frac{1}{2}\|\mathbf{Y}-\mathbf{A} \mathbf{X}\|_{F}^{2}+\lambda\|\mathbf{X}\|_{1,1} \\
\text { s.t. } \quad \mathbf{X} \geq 0,
\end{gathered}
$$

where $\|\mathbf{X}\|_{1,1}=\sum_{j=1}^{n}\left\|\mathbf{x}_{j}\right\|_{1}, \mathbf{x}_{j}$ is the $j$ th column vector of $\mathbf{X}$. The SUnSAL algorithm efficiently solves model (3) [19]. However, the $\ell_{1}$ norm measures the sparsity of each abundance vector independently instead of considering the joint sparsity of all pixels in the scene. The mixed norm $\ell_{2,1}$ norm is proposed to improve sparsity among the rows of $\mathbf{X}$ collaboratively. The corresponding optimization problem is described as

$$
\begin{gathered}
\min _{\mathbf{X}} \frac{1}{2}\|\mathbf{Y}-\mathbf{A} \mathbf{X}\|_{F}^{2}+\lambda\|\mathbf{X}\|_{2,1} \\
\text { s.t. } \quad \mathbf{X} \geq 0,
\end{gathered}
$$


where $\|\mathbf{X}\|_{2,1}=\sum_{i=1}^{m}\left\|\mathbf{x}_{i}\right\|_{2}, \mathbf{x}_{i}$ is the $i$ th row vector of $\mathbf{X}$. The optimization problem related to (4) is solved by the CLSUnSAL algorithm. As an improvement of the SUnSAL algorithm, it is aimed at finding a structured solution with only a few nonzero lines. In other words, it promotes the row sparsity of the abundance matrix $\mathbf{X}$.

The abovementioned unmixing models underutilize the spatial structure features of hyperspectral images. The rich spatial information contained in the image, which has an important impact on the unmixing performance, is not incorporated into these models. To this end, we propose a new sparse unmixing algorithm to address the aforementioned limitation.

\section{The Proposed Low-Rank and Spectral-Spatial Sparse Unmixing Algorithm}

3.1. The Proposed LRSSU Model. Inspired by the idea of iterative weighting [27], the spatial weight based on neighborhood is introduced into $\ell_{2,1}$ norm sparse regularization term, which integrates the spatial and spectral information. Due to the advantage of the low-rank representation model in maintaining the low-dimensional structure of the image, a low-rank regularization term is incorporated to explore the global spatial correlation of the hyperspectral data. Combining the sparse and low-rank constraints, a new sparse unmixing model with low-rank constraint is proposed as follows:

$$
\begin{gathered}
\min _{\mathbf{X}} \frac{1}{2}\|\mathbf{Y}-\mathbf{A X X}\|_{F}^{2}+\lambda\left\|\mathbf{H}_{\text {spa }} \odot \mathbf{X}\right\|_{2,1}+\tau \operatorname{rank}(\mathbf{X}) \\
\text { s.t. } \quad \mathbf{X} \geq 0,
\end{gathered}
$$

where $\mathbf{H}_{\text {spa }} \in \mathbb{R}^{m \times n}$ is the spatial weight matrix, $\odot$ is the Hadamard product, rank $(\mathbf{X})$ is the rank of the abundance matrix $\mathbf{X}$ , and $\lambda$ and $\tau$ are nonnegative regularization parameters. $\mathbf{H}_{\text {spa }}$ is updated iteratively as follows:

$$
\mathbf{H}_{\mathrm{spa}, i j}^{(t+1)}=\frac{\mathbf{1}}{f_{\mathcal{N}(p)}\left(x_{p}^{(t)}\right)+\varepsilon},
$$

where $\mathbf{H}_{\text {spa }, i j}^{(t)}$ represents the element in the $i$ th row and $j$ th column of $\mathbf{H}_{\text {spa }}$ at the $t$ th iteration. $p$ represents the pixel corresponding to fractional abundance $x_{i j}, x_{p}=\left(x_{1 j}, x_{2 j}, \cdots, x_{m j}\right)^{T}$ is the abundance vector for pixel $p, \mathcal{N}(p)$ represents the neighbor pixel set centered on $p$ in the image, and $f_{\mathcal{N}(\cdot)}(\cdot)$ denotes a function that explores the spatial correlation of pixels in a neighborhood system $\mathcal{N}(\cdot)$. In this paper, the 8 neighbor pixels of $p$ constitute the neighborhood system $\mathcal{N}_{8}(p)$; then, the function $f(\cdot)$ is defined as

$$
f_{\mathcal{N}_{8}(p)}\left(x_{p}\right)=\frac{\sum_{q \in \mathcal{N}_{8}(p)} \rho_{q} x_{q}}{\sum_{q \in \mathcal{N}_{8}(p)} \rho_{q}}
$$

where $q$ is any pixel in the 8 neighbor pixel set of $p, x_{q}$ is the abundance vector corresponding to $q, \rho_{q}=1 /\left(d_{E}(p, q)\right)$ denotes the neighborhood weight with regard to $q$, and $d_{E}(p$ ,$q$ ) denotes the Euclidean distance between pixel $q$ and its central pixel $p$.

The rank of $\mathbf{X}$ is closely relevant to the similarity of abundance vectors, and the reduced rank abundance matrix consists of fewer linearly independent vectors accordingly. When $\ell_{2,1}$ norm is used to promote the row sparsity of $\mathbf{X}$, the low-rank regularizer helps to determine the endmembers from the spectral library more accurately. Nevertheless, it is difficult to calculate the rank of the matrix directly. The nuclear norm $\|\mathbf{X}\|_{*}$ is the tightest convex relaxation of the rank of $\mathbf{X}$. Therefore, we replace $\operatorname{rank}(\mathbf{X})$ with $\|\mathbf{X}\|_{*}$ and rewrite model (5) as

$$
\begin{gathered}
\min _{\mathbf{X}} \frac{1}{2}\|\mathbf{Y}-\mathbf{A X}\|_{F}^{2}+\lambda\left\|\mathbf{H}_{\text {spa }} \odot \mathbf{X}\right\|_{2,1}+\tau\|\mathbf{X}\|_{*} \\
\text { s.t. } \quad \mathbf{X} \geq 0,
\end{gathered}
$$

where $\|\mathbf{X}\|_{*}=\sum_{i} \sigma_{i}(\mathbf{X})$ and $\sigma_{i}(\mathbf{X})$ is the $i$ th singular value of $\mathrm{X}$.

The weighted nuclear norm minimization (WNNM) can enhance the sparsity of the singular vector by imposing different weights on each singular value [28]. Specifically, smaller singular values that carry less valid information are assigned larger weights and then are greatly reduced or even reduced to zero. On the contrary, larger values are assigned smaller weights, so that the main information is well preserved. The weighted nuclear norm $\|\mathbf{X}\|_{\mathbf{b}, *}$ is adopted to improve the robustness and consistency of model (8), which is expressed as

$$
\|\mathbf{X}\|_{\mathbf{b}, *}=\sum_{i=1}^{r} b_{i} \sigma_{i}(\mathbf{X})
$$

where $\mathbf{b}=\left(b_{1}, b_{2}, \cdots\right)$ is the nonnegative weight vector and $b_{i}$ is the weight related to the $i$ th singular value of the matrix $\mathbf{X}$. At the $k$ th iteration, $b_{i}$ is updated as follows:

$$
b_{i}^{(k)}=\frac{1}{\sigma_{i}\left(\mathbf{X}^{(k)}\right)+\varepsilon},
$$

where $\varepsilon>0$ is a small constant added to avoid singularity and $\mathbf{X}^{(k)}$ represents the abundance matrix $\mathbf{X}$ at the $k$ th iteration.

In summary, the low-rank and spectral-spatial sparse unmixing model (LRSSU) is expressed as follows:

$$
\begin{gathered}
\min _{\mathbf{X}} \frac{1}{2}\|\mathbf{Y}-\mathbf{A X}\|_{F}^{2}+\lambda\left\|\mathbf{H}_{\text {spa }} \odot \mathbf{X}\right\|_{2,1}+\tau\|\mathbf{X}\|_{\mathbf{b}, *} \\
\text { s.t. } \quad \mathbf{X} \geq 0 .
\end{gathered}
$$

3.2. Solution of the Optimization Problem. In this section, an iterative optimization scheme of internal and external dual circulation based on the alternating direction method of multipliers (ADMM) [29] is proposed. The external 
circulation updates the spatial weight matrix following equation (6), and the internal circulation solves the abundance matrix in the framework of ADMM. The flow of solving model (11) is described in detail as follows.

The auxiliary matrix variables $\mathbf{U}, \mathbf{V}_{1}, \mathbf{V}_{2}, \mathbf{V}_{3}$, and $\mathbf{V}_{4}$ are introdued, and model (11) is equivalently converted to the following form:

$$
\begin{gathered}
\min _{\mathbf{U}, \mathbf{V}_{1}, \mathbf{V}_{2}, \mathbf{V}_{3}, \mathbf{V}_{4}} \frac{1}{2}\left\|\mathbf{Y}-\mathbf{V}_{1}\right\|_{F}^{2}+\lambda\left\|\mathbf{H}_{\text {spa }} \odot \mathbf{V}_{2}\right\|_{2,1}+\tau\left\|\mathbf{V}_{3}\right\|_{\mathbf{b}, *}+\iota_{R+}\left(\mathbf{V}_{4}\right) \\
\text { s.t. } \quad \mathbf{U}=\mathbf{X} \\
\mathbf{V}_{1}=\mathbf{A} \mathbf{U} \\
\mathbf{V}_{2}=\mathbf{U} \\
\mathbf{V}_{3}=\mathbf{U} \\
\mathbf{V}_{4}=\mathbf{U}
\end{gathered}
$$

where $\iota_{R+}(\mathbf{X})=\sum_{i=1}^{n} \iota_{R+}\left(\mathbf{x}_{i}\right)$ is the indicator function; i.e., $\iota_{R+}$ $\left(\mathbf{x}_{i}\right)$ is zero if $\mathbf{x}_{i}$ is nonnegative and $+\infty$ otherwise. Equation (12) can be expressed compactly as

$$
\min _{\mathbf{U}, \mathbf{V}} g(\mathbf{U}, \mathbf{V})
$$$$
\text { s.t. } \mathbf{G U}+\mathbf{B V}=0 \text {, }
$$

where $\quad g(\mathbf{U}, \mathbf{V})=(1 / 2)\left\|\mathbf{Y}-\mathbf{V}_{1}\right\|_{F}^{2}+\lambda\left\|\mathbf{H}_{\text {spa }} \odot \mathbf{V}_{2}\right\|_{2,1}+\tau$ $\left\|\mathbf{V}_{3}\right\|_{\mathbf{b}, *}+\iota_{R+}\left(\mathbf{V}_{4}\right), \mathbf{G}=(\mathbf{A}, \mathbf{I}, \mathbf{I}, \mathbf{I})^{\mathbf{T}}, \mathbf{B}=\operatorname{diag}(-I)$, and $\mathbf{V}=$ $\left(\mathbf{V}_{1}, \mathbf{V}_{2}, \mathbf{V}_{3}, \mathbf{V}_{4}\right)^{\mathbf{T}}$

The augmented Lagrangian of equation (13) is written as

$$
\mathscr{L}(\mathbf{U}, \mathbf{V}, \mathbf{D})=g(\mathbf{U}, \mathbf{V})+\frac{\mu}{2}\|\mathbf{G} \mathbf{U}+\mathbf{B V}-\mathbf{D}\|_{F}^{2},
$$

where $\mu$ is a nonnegative penalty parameter, $\mathbf{D}=$ $\left(\mathbf{D}_{1}, \mathbf{D}_{2}, \mathbf{D}_{3}, \mathbf{D}_{4}\right)^{\mathbf{T}}$ is the Lagrange multipliers with regards to $\mathbf{G U}+\mathbf{B V}=0$.

The optimization problem (13) can be solved by minimizing $\mathscr{L}(\mathbf{U}, \mathbf{V}, \mathbf{D})$ in the framework of ADMM. We update the variables $\mathbf{U}$ and $\mathbf{V}$ sequentially by solving the following subproblems:

$$
\left\{\begin{array}{l}
\mathbf{U}^{(k+1)}=\arg \min _{\mathbf{U}} \mathscr{L}\left(\mathbf{U}, \mathbf{V}^{(k)}, \mathbf{D}^{(k)}\right), \\
\mathbf{V}^{(k+1)}=\arg \min _{\mathbf{V}} \mathscr{L}\left(\mathbf{U}^{(k+1)}, \mathbf{V}, \mathbf{D}^{(k)}\right) .
\end{array}\right.
$$

The U-subproblem has the closed-form solution, which is obtained by taking the partial derivative of $\mathbf{U}$. The variable $\mathbf{U}$ is calculated as follows:

$$
\begin{aligned}
\mathbf{U}^{(k+1)}= & \arg \min _{\mathbf{U}} \frac{\mu}{2}\left\|\mathbf{A} \mathbf{U}-\mathbf{V}_{1}^{(k)}-\mathbf{D}_{1}^{(k)}\right\|_{F}^{2} \\
& +\frac{\mu}{2}\left\|\mathbf{U}-\mathbf{V}_{2}^{(k)}-\mathbf{D}_{2}^{(k)}\right\|_{F}^{2}+\frac{\mu}{2}\left\|\mathbf{U}-\mathbf{V}_{3}^{(k)}-\mathbf{D}_{3}^{(k)}\right\|_{F}^{2} \\
& +\frac{\mu}{2}\left\|\mathbf{U}-\mathbf{V}_{4}^{(k)}-\mathbf{D}_{4}^{(k)}\right\|_{F}^{2} \\
= & \left(\mathbf{A}^{\mathbf{T}} \mathbf{A}+3 \mathbf{I}\right)^{-1}\left(\mathbf{A}^{\mathbf{T}}\left(\mathbf{V}_{1}^{(k)}+\mathbf{D}_{1}^{(k)}\right)+\mathbf{V}_{2}^{(k)}+\mathbf{D}_{2}^{(k)}\right. \\
& \left.+\mathbf{V}_{3}^{(k)}+\mathbf{D}_{3}^{(k)}+\mathbf{V}_{4}^{(k)}+\mathbf{D}_{4}^{(k)}\right) .
\end{aligned}
$$

The $\mathbf{V}$-subproblem is split into four independent optimization problems with regard to $\mathbf{V}_{1}, \mathbf{V}_{2}, \mathbf{V}_{3}$, and $\mathbf{V}_{4}$. The solution of $\mathbf{V}_{1}$ is calculated as follows:

$$
\begin{aligned}
\mathbf{V}_{1}^{(k+1)} & =\arg \min _{\mathbf{V}_{1}} \frac{1}{2}\left\|\mathbf{Y}-\mathbf{V}_{1}\right\|_{F}^{2}+\frac{\mu}{2}\left\|\mathbf{A} \mathbf{U}^{(k+1)}-\mathbf{V}_{1}-\mathbf{D}_{1}^{(k)}\right\|_{F}^{2} \\
& =\frac{1}{1+\mu}\left[\mathbf{Y}+\mu\left(\mathbf{A} \mathbf{U}^{(k+1)}-\mathbf{D}_{1}^{(k)}\right)\right] .
\end{aligned}
$$

The optimization problem related to $\mathbf{V}_{2}$ is

$$
\mathbf{V}_{2}^{(k+1)}=\arg \min _{\mathbf{V}_{2}} \lambda\left\|\mathbf{H}_{\text {spa }} \odot \mathbf{V}_{2}\right\|_{2,1}+\frac{\mu}{2}\left\|\mathbf{U}^{(k+1)}-\mathbf{V}_{2}-\mathbf{D}_{2}^{(k)}\right\|_{F}^{2}
$$

The $r$ th row of $\mathbf{V}_{2}$ is written as $\mathbf{V}_{2, r}$; then, the closedform solution of problem (18) is

$$
\mathbf{V}_{2, r}^{(k+1)}=\operatorname{vect}-\mathbf{s o f t}\left(\mathbf{U}_{r}^{(k+1)}-\mathbf{D}_{2, r}^{(k)}, \frac{\lambda}{\mu} \mathbf{H}_{\mathrm{spa}, r}\right)
$$

where vect-soft $(y, \tau)=y\left(\max \left\{\|y\|_{2}-\tau, 0\right\} / \max \left\{\|y\|_{2}-\tau\right.\right.$, $0\}+\tau)$ denotes the soft threshold function for row vector. $\mathbf{V}_{3}$ is updated according to the following optimization problem:

$$
\begin{aligned}
\mathbf{V}_{3}^{(k+1)} & =\arg \min _{\mathbf{V}_{3}} \tau\left\|\mathbf{V}_{3}\right\|_{\mathbf{b}, *}+\frac{\mu}{2}\left\|\mathbf{U}^{(k+1)}-\mathbf{V}_{3}-\mathbf{D}_{3}^{(k)}\right\|_{F}^{2} \\
& =\mathbf{S V T}_{\mathbf{b}, \tau / \mu}\left(\mathbf{U}^{(k+1)}-\mathbf{D}_{3}^{(k)}\right),
\end{aligned}
$$

where $\operatorname{SVT}_{\mathbf{b}, \omega}(\cdot)$ denotes the singular value threshold function and the weight vector $\mathbf{b}$ is calculated according to equation (10). The singular value decomposition (SVD) of $\mathbf{X}$ is written as $\mathbf{X}=\widehat{\mathbf{U D i a g}}\left(\sigma_{1}, \cdots, \sigma_{r}\right) \mathbf{V} \wedge^{\mathbf{T}}$, then $\mathbf{S V T}_{\mathbf{b}, \omega}(\mathbf{X})=\widehat{\mathbf{U D}}$ $\operatorname{iag}\left(\max \left(\sigma_{1}-\omega b_{1}, 0\right)\right), \cdots,\left(\max \left(\sigma_{r}-\omega b_{r}, 0\right)\right) \mathbf{V} \wedge^{\mathrm{T}}$. In the same vein, we get the solution of $\mathbf{V}_{4}$ as follows:

$$
\begin{aligned}
\mathbf{V}_{4}^{(k+1)} & =\arg \min _{\mathbf{V}_{4}} \iota_{R+}\left(\mathbf{V}_{4}\right)+\frac{\mu}{2}\left\|\mathbf{U}^{(k+1)}-\mathbf{V}_{4}-\mathbf{D}_{4}^{(k)}\right\|_{F}^{2} \\
& =\max \left(\mathbf{U}^{(k+1)}-\mathbf{D}_{4}^{(k)}, 0\right) .
\end{aligned}
$$




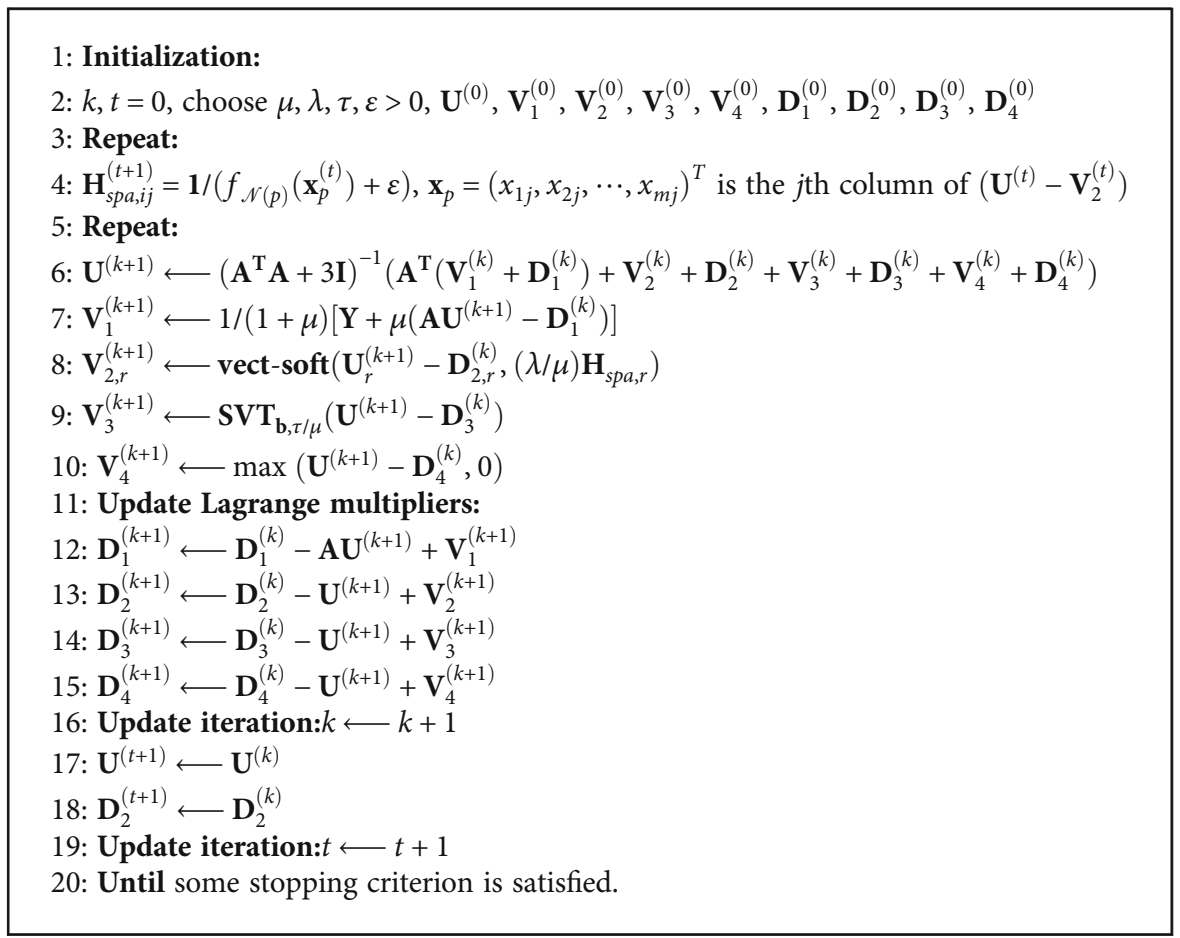

Algorithm 1: Pseudocode of the LRSSU algorithm.

Finally, the Lagrange multipliers are updated as follows:

$$
\mathbf{D}^{(k+1)}=\mathbf{D}^{(k)}-\left(\mathbf{G} \mathbf{U}^{(k+1)}+\mathbf{B} \mathbf{V}^{(k+1)}\right)
$$

To sum up, the complete scheme for the proposed LRSSU model is presented in Algorithm 1, which consists of internal and external dual circulations. The external circulation updates the spatial weight matrix in step 4, which is set up to 100 iterations. The internal circulation updates the variables and Lagrange multipliers in ADMM framework from step 6 to step 15 and sets 5 iterations empirically $[10,30,31]$.

The convergence of Algorithm 1 is difficult to prove. Nevertheless, the residual of the optimization problem decreases with the increase of iterations. Figure 1 shows the residual $\left\|\mathbf{G} \mathbf{U}^{(t)}+\mathbf{B} \mathbf{V}^{(t)}\right\|_{F}$ as a function of the number of iterations for the complete algorithm. It can be observed that the residual arrived at a certain level close to zero after about 20 iterations of the external circulation. Therefore, the stopping criterion of the algorithm is set as the residual error reaches the error tolerance or the number of iterations reaches the upper limit, which guarantees the convergence of the algorithm.

\section{Experimental Analysis}

In this section, the performance of the proposed LRSSU algorithm will be discussed in simulated and real hyperspectral data experiments. In the simulated data experiment, the signal-to-reconstruction error (SRE) is used to quantitatively

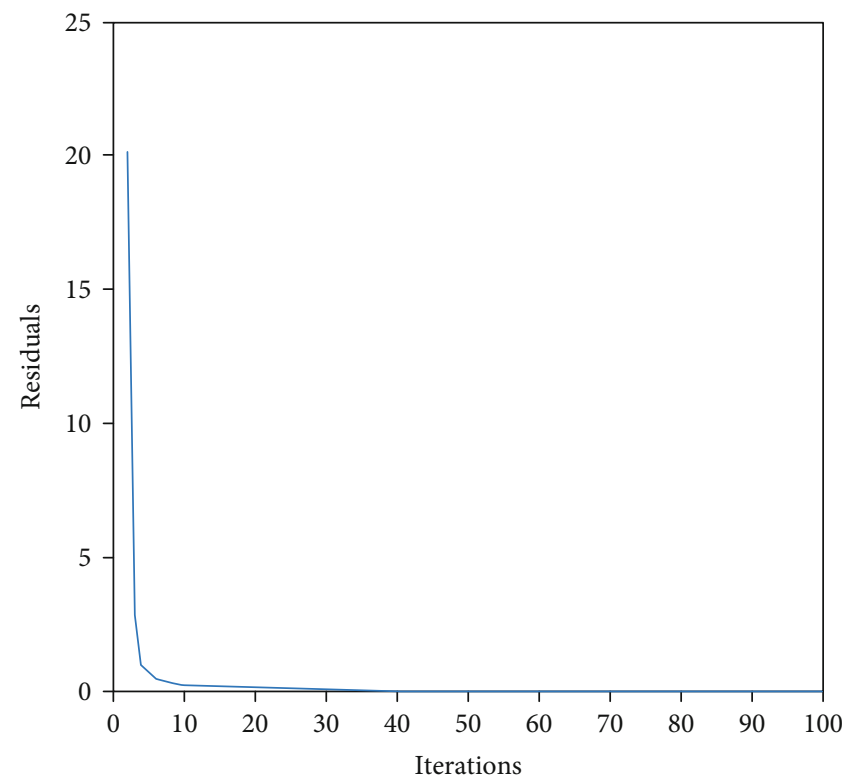

Figure 1: The residual $\left\|\mathbf{G} \mathbf{U}^{(t)}+\mathbf{B} \mathbf{V}^{(t)}\right\|_{F}$ as a function of the number of iterations for the complete algorithm.

evaluate the unmixing accuracy. The SRE $(\mathrm{dB})$ is defined as follows:

$$
\operatorname{SRE}(\mathrm{dB})=10 \cdot \log _{10}\left(E\left(\|\mathbf{x}\|_{2}^{2}\right) / E\left(\|\mathbf{x}-\widehat{\mathbf{x}}\|_{2}^{2}\right)\right)
$$

where $E(\cdot)$ is the expectation function, $\widehat{\mathbf{x}}$ is the estimated abundance vector, $\mathbf{x}$ is the true abundance vector. In addition, the probability of success $p_{s}$ is another quantitative 

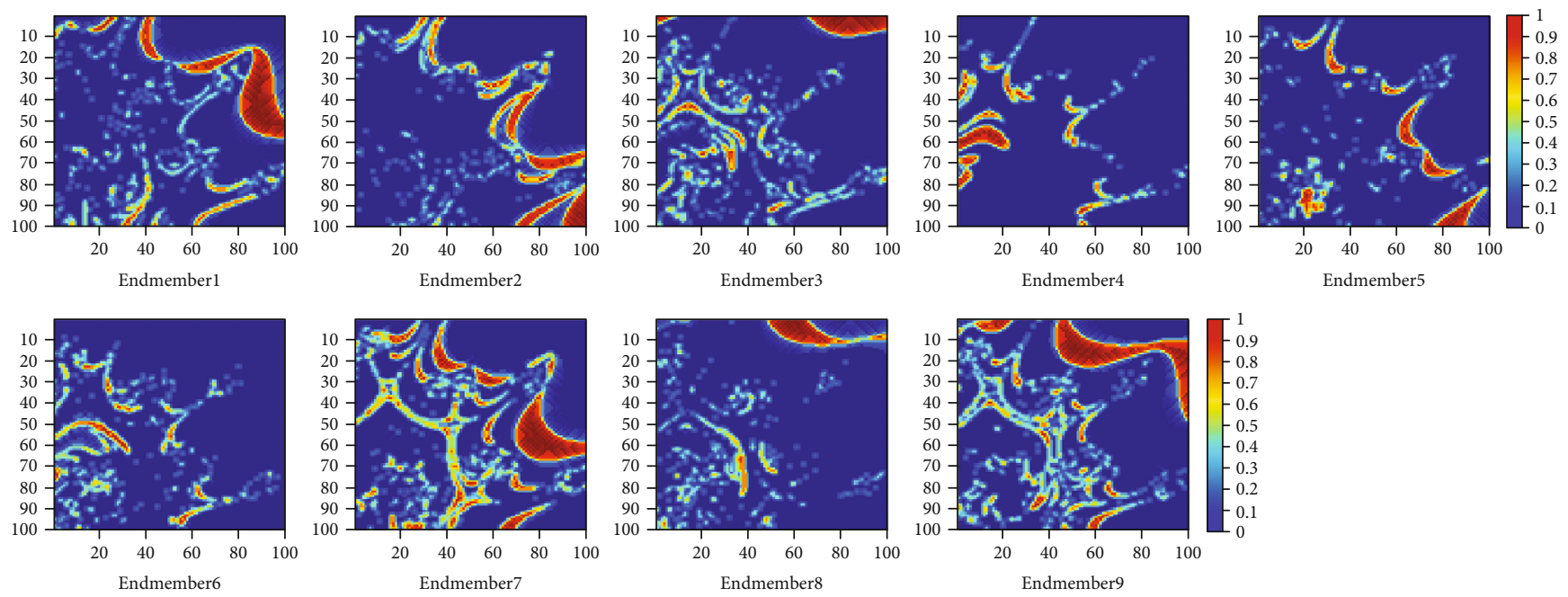

FIGURE 2: True fractional abundances of the endmembers in the simulated data.

TABLE 1: SRE (B) and $p_{s}$ results after applying different unmixing algorithms to the simulated data (the optimal parameter settings for which the reported values were achieved are shown in the parentheses).

\begin{tabular}{|c|c|c|c|c|c|c|}
\hline \multirow{2}{*}{ Algorithm } & \multicolumn{2}{|c|}{$\mathrm{SNR}=30 \mathrm{~dB}$} & \multicolumn{2}{|c|}{$\mathrm{SNR}=40 \mathrm{~dB}$} & \multicolumn{2}{|c|}{$\mathrm{SNR}=50 \mathrm{~dB}$} \\
\hline & SRE $(\mathrm{dB})$ & $p_{s}$ & SRE $(\mathrm{dB})$ & $p_{s}$ & SRE (dB) & $p_{s}$ \\
\hline \multirow{2}{*}{ SUnSAL } & 6.3967 & 0.6303 & 11.5562 & 0.8871 & 18.9222 & 0.9991 \\
\hline & \multicolumn{2}{|c|}{$(\lambda=8 e-3)$} & \multicolumn{2}{|c|}{$(\lambda=2 e-3)$} & \multicolumn{2}{|c|}{$(\lambda=3 e-4)$} \\
\hline \multirow{2}{*}{ CLSUnSAL } & 6.6157 & 0.7269 & 14.7819 & 0.9999 & 26.6827 & 1 \\
\hline & \multicolumn{2}{|c|}{$(\lambda=3 e-1)$} & \multicolumn{2}{|c|}{$(\lambda=2 e-2)$} & \multicolumn{2}{|c|}{$(\lambda=7 e-3)$} \\
\hline \multirow{2}{*}{ SUnSAL-TV } & 9.0378 & 0.7822 & 15.4433 & 0.9861 & 25.3656 & 1 \\
\hline & \multicolumn{2}{|c|}{$\left(\lambda=4 e-3 ; \lambda_{\mathrm{TV}}=2 e-3\right)$} & \multicolumn{2}{|c|}{$\left(\lambda=6 e-5 ; \lambda_{\mathrm{TV}}=9 e-4\right)$} & \multicolumn{2}{|c|}{$\left(\lambda=5 e-5 ; \lambda_{\mathrm{TV}}=9 e-5\right)$} \\
\hline \multirow{2}{*}{ DRSU } & 14.3296 & 0.9465 & 26.0656 & 1 & 34.4967 & 1 \\
\hline & \multicolumn{2}{|c|}{$(\lambda=2 e-3)$} & \multicolumn{2}{|c|}{$(\lambda=6 e-4)$} & \multicolumn{2}{|c|}{$(\lambda=1 e-4)$} \\
\hline \multirow{2}{*}{ JSpBLRU } & 15.0546 & 0.9928 & 24.8702 & 1 & 34.0073 & 1 \\
\hline & \multicolumn{2}{|c|}{$(\lambda=2.3 ; \tau=1.4)$} & \multicolumn{2}{|c|}{$(\lambda=4 e-1 ; \tau=2 e-1)$} & \multicolumn{2}{|c|}{$(\lambda=2 e-1 ; \tau=4 e-2)$} \\
\hline \multirow{2}{*}{ LRSSU } & 19.8380 & 0.9989 & 27.9738 & 1 & 36.4791 & 1 \\
\hline & \multicolumn{2}{|c|}{$(\lambda=4 e-2 ; \tau=8 e-2)$} & \multicolumn{2}{|c|}{$(\lambda=2 e-2 ; \tau=1 e-2)$} & \multicolumn{2}{|c|}{$(\lambda=4 e-3 ; \tau=2 e-3)$} \\
\hline
\end{tabular}

evaluation indicator, which appraises the stability of the results [5] . $p_{s}$ is defined as follows: $p_{s} \equiv P\left(\|\mathbf{x} \wedge-\mathbf{x}\|^{2} /\|\mathbf{x}\|^{2}\right.$ $\leq$ threshold). The results will be considered successful, if the relative error power of results is less than an appropriate threshold, usually $3.16(5 \mathrm{~dB})$. The larger the SRE (dB) or $p_{s}$, the better the performance of the algorithm. For fair comparison, the parameters of all algorithms in the experiment are tuned to the optimal in advance, and the maximum number of iterations is set to 500. The proposed LRSSU algorithm is compared with the classic SUnSAL, CLSUnSAL, and SUnSAL-TV algorithms, as well as the advanced DRSU algorithm and the JSPBLRU algorithm considering the low-rank constraint.

4.1. Experiment on Simulated Data. The spectral library A $\in \mathbb{R}^{221 \times 222}$ used in the simulated experiment is composed of 222 spectral curves randomly selected from the splib06 spectral library provided by the United States Geological Survey (USGS) (http://speclab.cr.usgs.gov/spectral.lib06.), with 221 spectral bands. The reflectance values are evenly distributed in the range of $0.4-2.5 \mu \mathrm{m}$. The abundance map containing $100 \times 100$ pixels is generated form the HyperMix standard dataset, where ANC and ASC are implemented. The data imitates the distribution features of surface objects in nature, such as surface water, coastline, vegetation, and mountains, so it is widely used to test the unmixing performance of different algorithms. The simulated data cube is synthesized by nine endmembers selected from the library A, and then, white Gaussian noise with different signal-tonoise ratios (SNR) is added. The true fractional abundances of the nine endmembers are displayed in Figure 2.

Table 1 lists the value of SRE $(\mathrm{dB})$ and $p_{s}$ obtained by different algorithms on the simulated data at $\mathrm{SNR}=30 \mathrm{~dB}$, $40 \mathrm{~dB}$, and $50 \mathrm{~dB}$. From Table 1, we observe that the proposed LRSSU algorithm achieves higher SRE $(\mathrm{dB})$ and $p_{s}$ than other comparison algorithms at all noise levels. Compared with SUnSAL, CLSUnSAL, and SUnSAL-TV, the LRSSU algorithm shows great advantages in stability and 

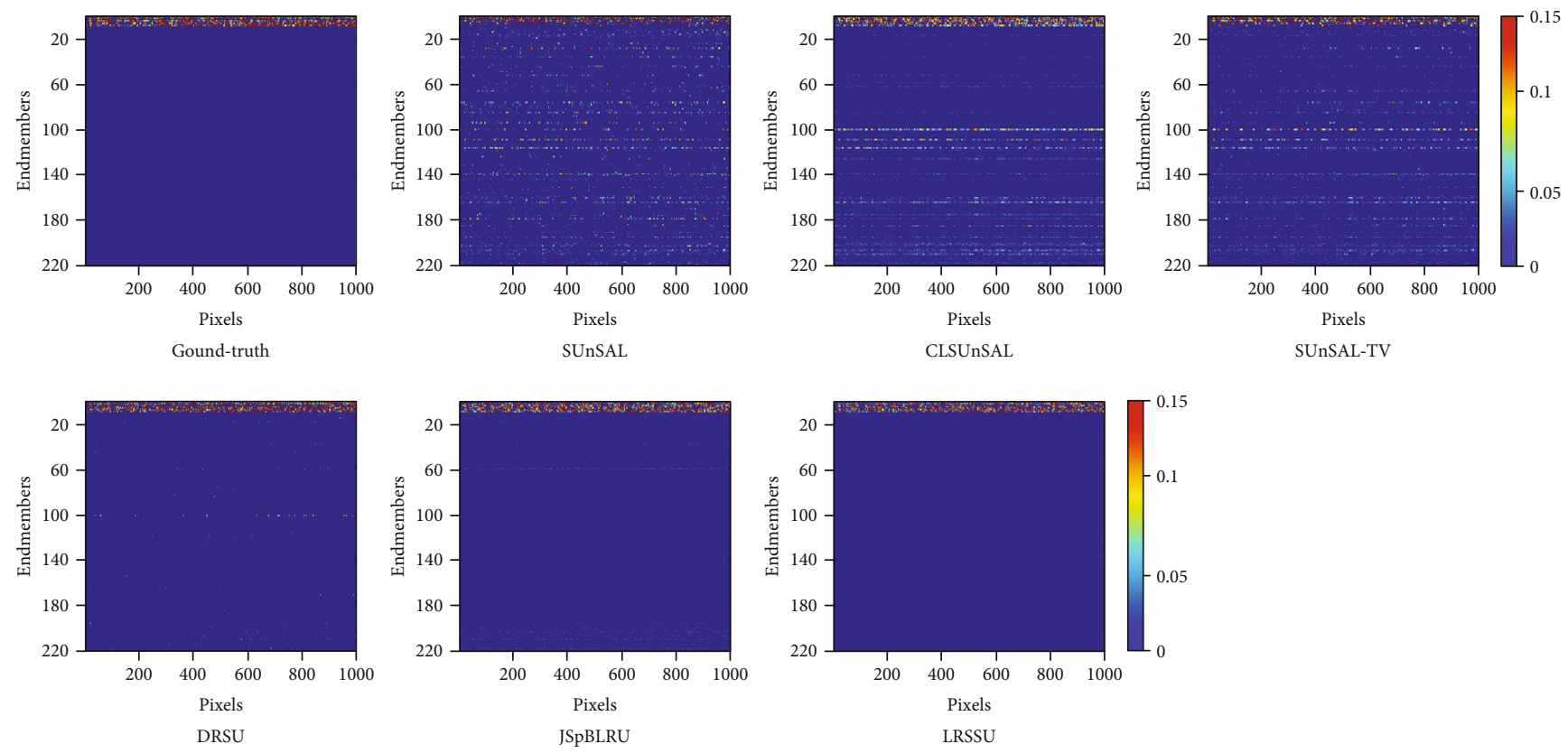

FIGURE 3: The ground-truth abundance and the estimated abundance maps obtained from the simulated data under noise ratio of SNR $=30$ dB.

accuracy, which proves its superior unmixing performance. In the case of high noise, the LRSSU and JSPBLRU algorithms maintain high $p_{s}$, which indicates that minimizing the rank of the abundance matrix can effectively improve the robustness of the unmixing algorithm. The SRE (dB) obtained by the LRSSU algorithm is higher than that of the DRSU and JSpBLRU algorithms, which demonstrates that the local spatial weighting strategy plays an important role in improving the accuracy of unmixing.

To illustrate the effect of the proposed LRSSU algorithm on endmember identification, Figure 3 exhibits estimated abundances obtained by each algorithm on the simulated data with $\mathrm{SNR}=30 \mathrm{~dB}$. For visual clarity, Figure 3 displays only 1000 pixels selected from the image. Each colored line in the figure represents a nonzero row vector in the abundance matrix, corresponding to an actual endmember. It can be seen from the figure that the abundance map obtained by the LRSSU algorithm is consistent with the ground-truth abundance. More specifically, the endmembers identified by the LRSSU algorithm from the spectral library are the same as the true ones, with the least outliers. The abundance maps estimated by the SUnSAL, CLSUnSAL, and SUnSAL-TV algorithms emerge a mass of false endmember lines and interference noises, which do not match the actual endmembers well. The DRSU algorithm confuses some actual endmembers with other highly similar entries in the spectral library, resulting in endmember mismatches, and it is susceptible to noise. The results obtained by the JSpBLRU algorithm are disturbed by abundance vectors with small values corresponding to false endmembers. It can be concluded that the proposed LRSSU algorithm further enhances the row sparsity of the abundance matrix and improves the accuracy of identifying endmembers from the spectral library.
Figure 4 exhibits the abundance maps of the ninth endmember estimated by different algorithms from the simulated data at $\mathrm{SNR}=30 \mathrm{~dB}$. The other endmembers exhibit similar behavior, so they are not shown. For a more intuitive comparison, Figure 4 also shows the difference maps between the estimated abundance obtained by each algorithm and the true abundance. The abundance map obtained by the proposed LRSSU algorithm is close to the true distribution, which reveals a significant advantage than other algorithms. The SUnSAL, CLSUnSAL, and SUnSALTV algorithms estimate the abundances inaccurately, and the unmixing results are obviously different from the true abundance map. Compared with the DRSU and JSpBLRU algorithms, the LRSSU algorithm retains more spatial detail information of the abundance map, showing better spatial smoothness and less noise. It is proved that the combination of spectral-spatial collaborative sparse and low-rank constraints enhances the sparsity of the solution, preserves the finer structure and texture information of the image, promotes the spatial consistency between pixels, and improves the accuracy of unmixing.

The setting of regularization parameters has an important impact on the unmixing performance of the algorithm. To analyze the sensitivity of parameters in the proposed LRSSU algorithm, Figure 5 shows the SRE (dB) as functions of parameters $\lambda$ and $\tau$. We observe that when the noise level decreases, the value of the optimal parameter decreases accordingly. Moreover, the parameter $\lambda$ affects the accuracy of solutions more than the parameter $\tau$. Overall, when the regularization parameters $\lambda$ and $\tau$ vary in a large range, SRE (dB) is still optimal or suboptimal. This is helpful to achieve the best performance of the algorithm and improve its practical application. 

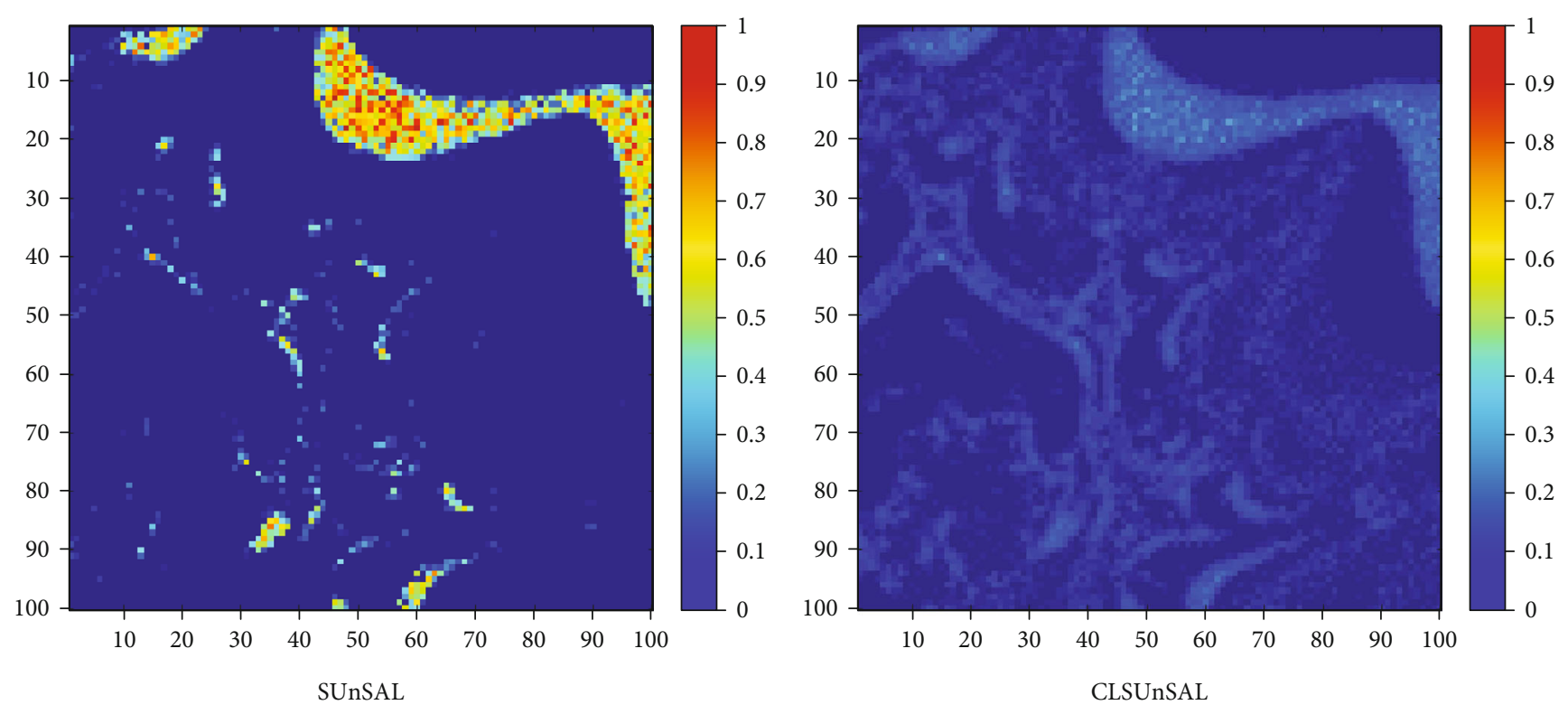

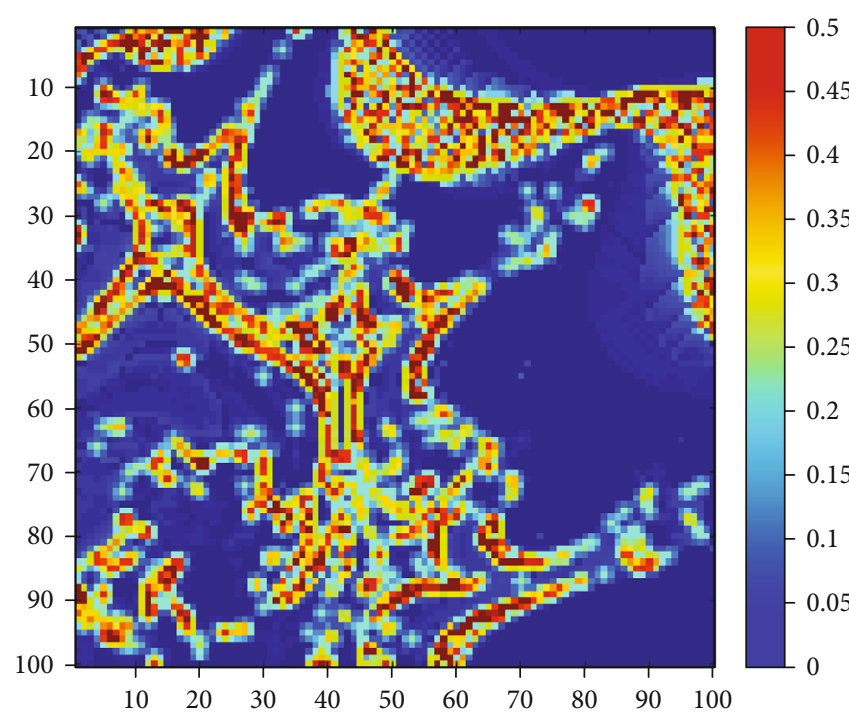

(a)

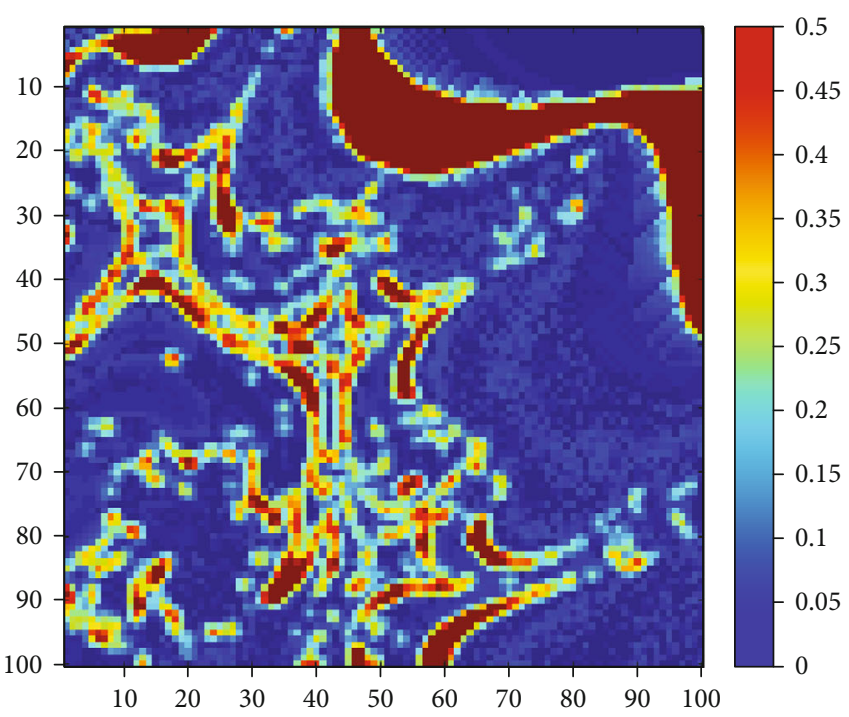

(b)

FIgURE 4: Continued. 

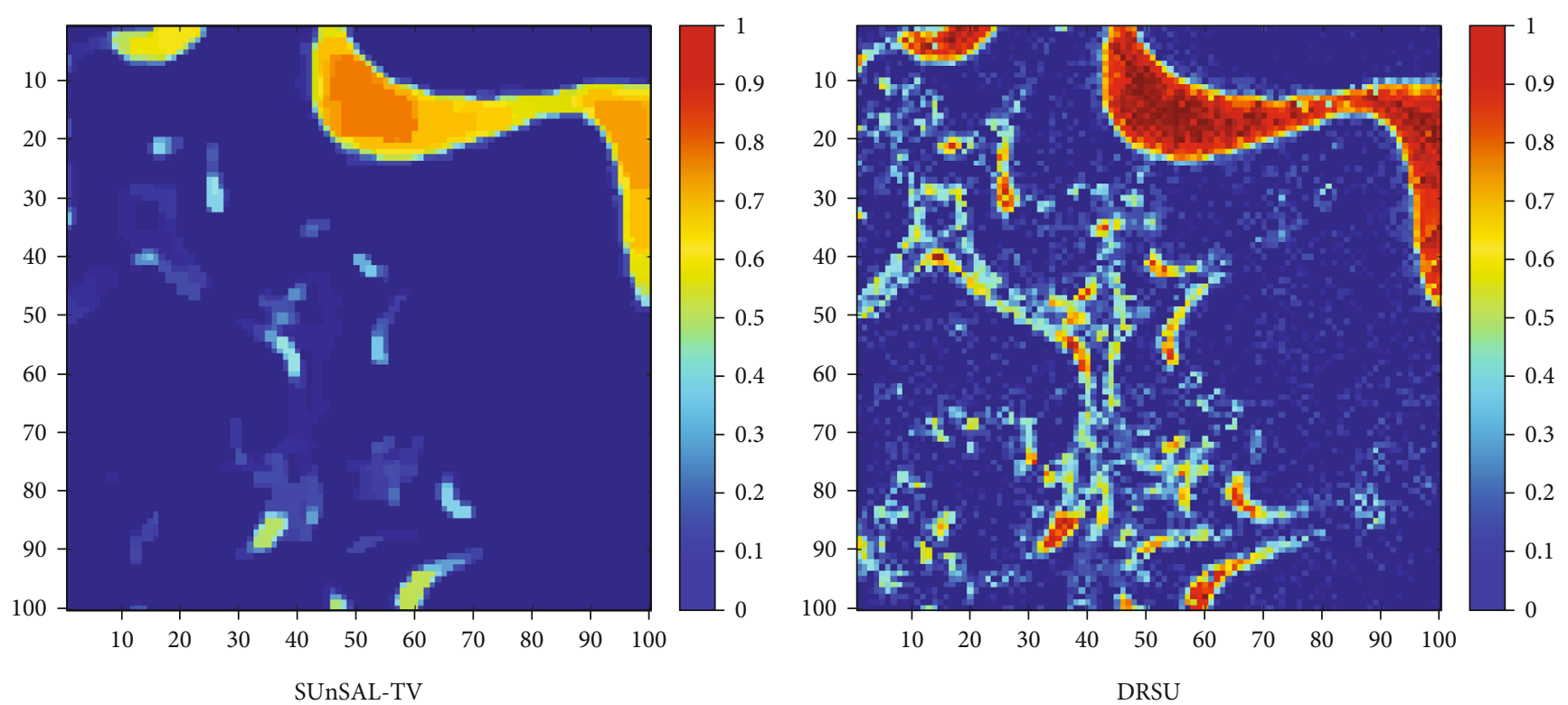

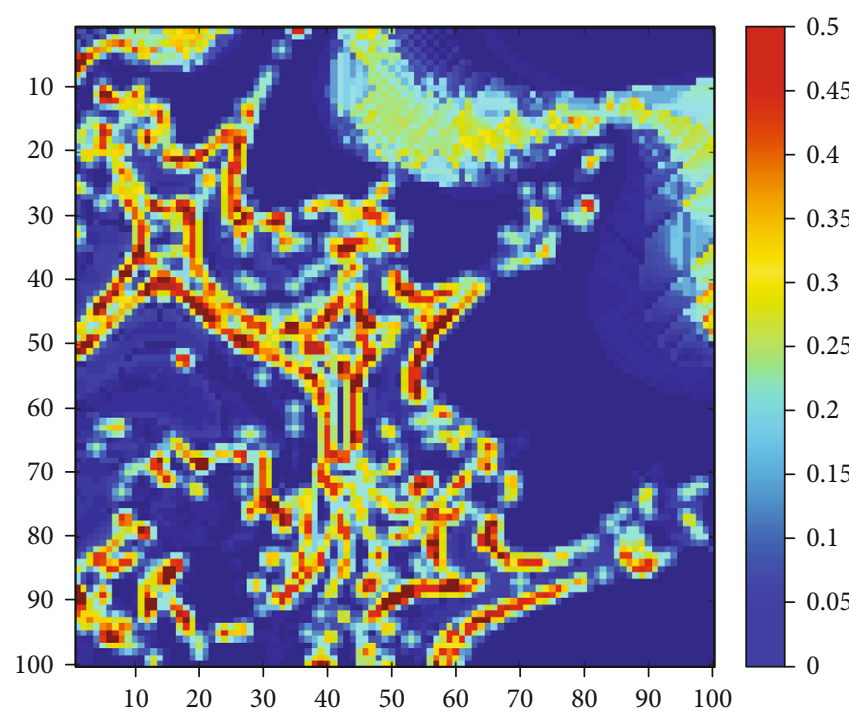

(c)

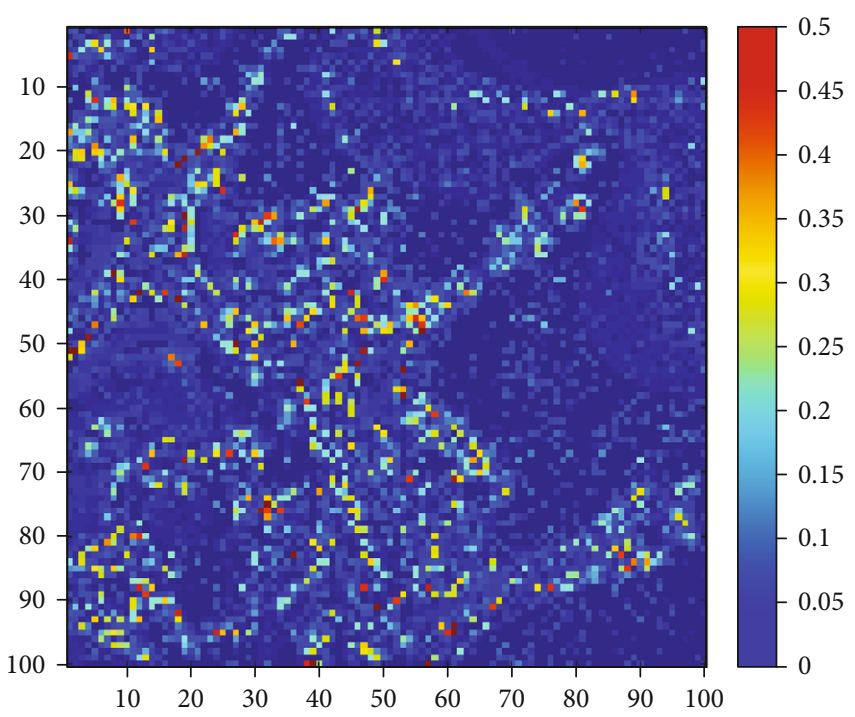

(d)

Figure 4: Continued. 

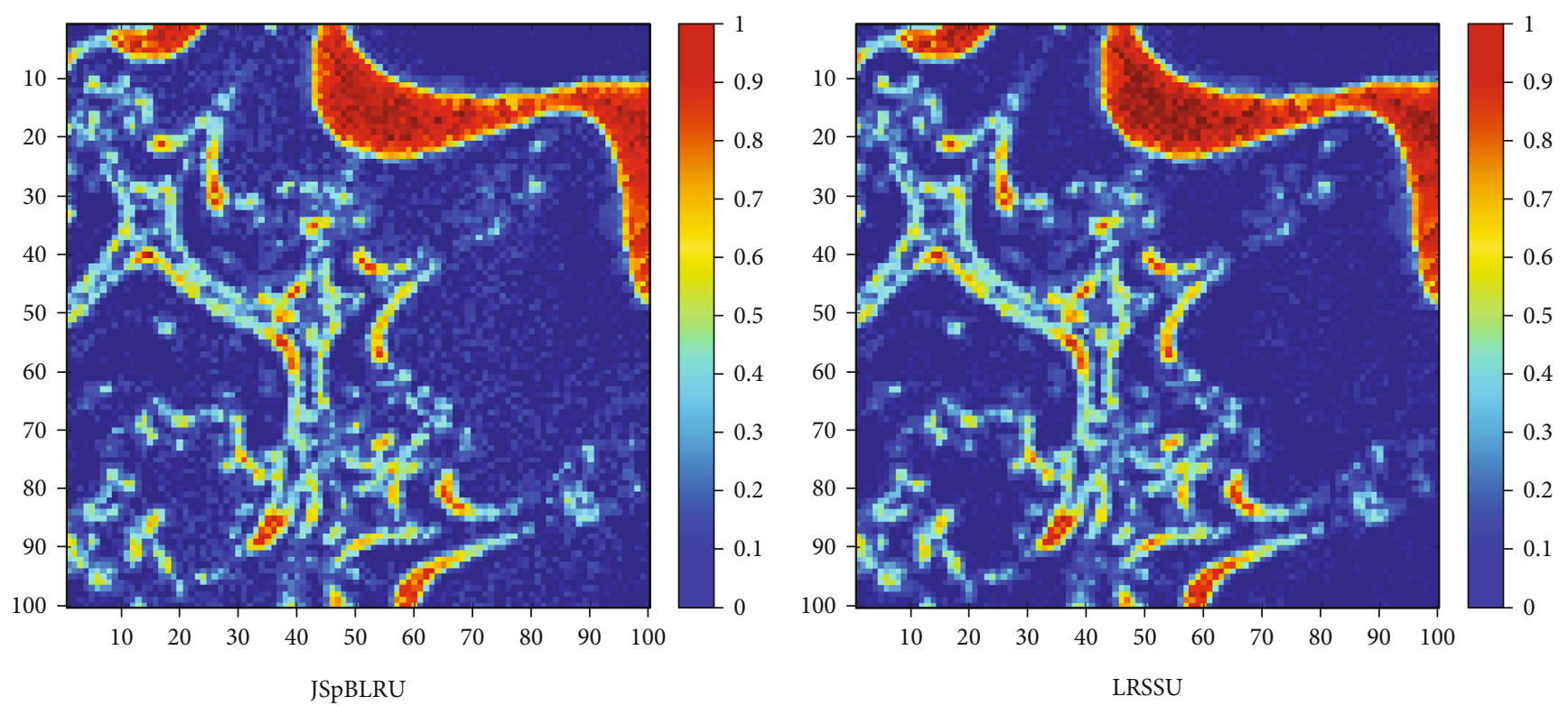

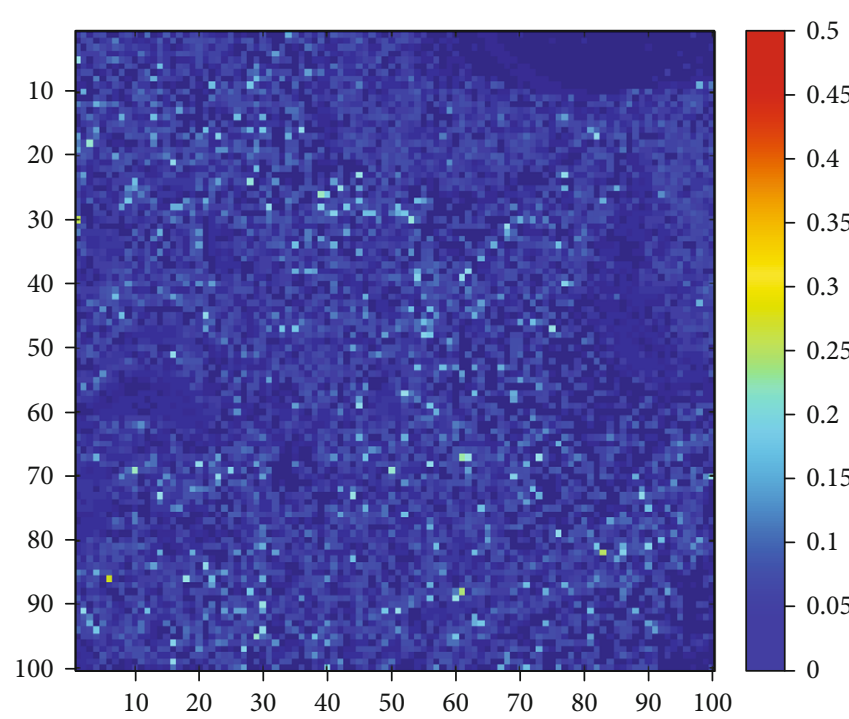

(e)

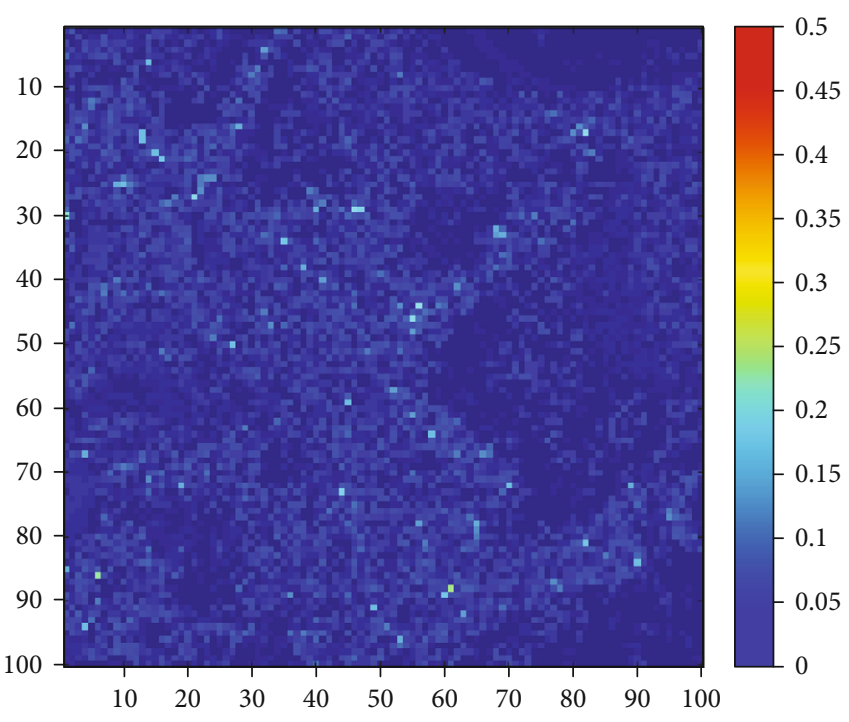

(f)

Figure 4: Abundance maps obtained for the ninth endmember from the simulated data under noise ratio of SNR $=30 \mathrm{~dB}$. Difference maps between the ground-truth abundance and the results obtained by (a) SUnSAL, (b) CLSUnSAL, (c) SUnSAL-TV, (d) DRSU, (e) JSpBLRU, and (f) LRSSU. 

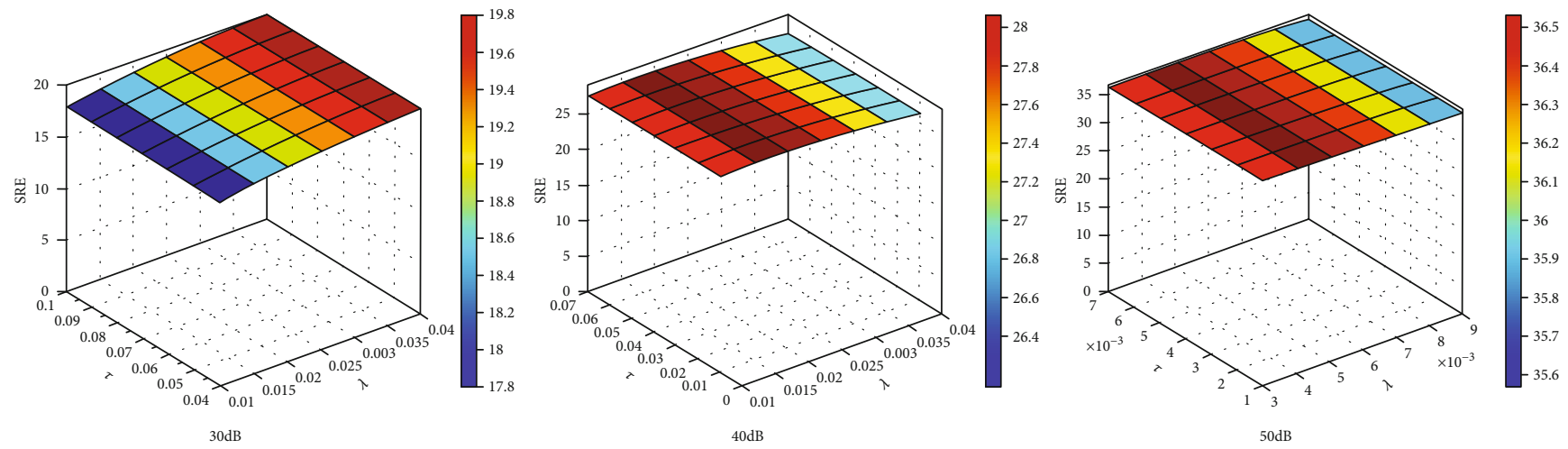

FIGURE 5: SRE (B) as functions of parameters $\lambda$ and $\tau$ for the simulated data at $\mathrm{SNR}=30 \mathrm{~dB}$.
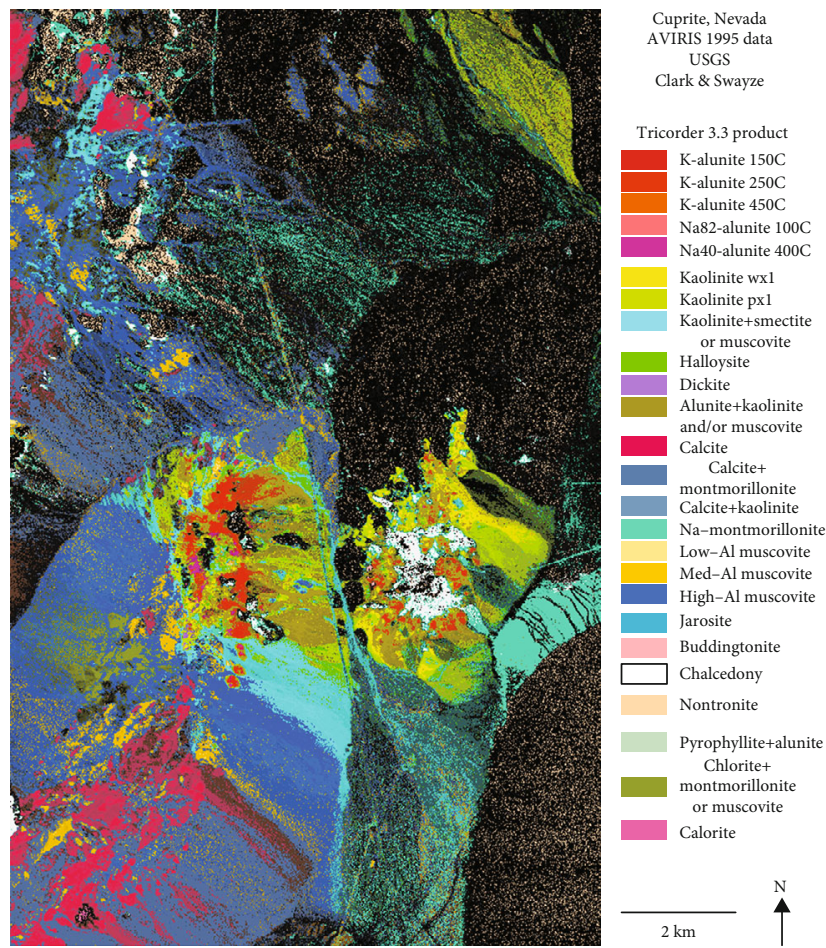

FIGURE 6: USGS mineral map of Cuprite mining district in Nevada [5].

4.2. Experiment on Real Data. The Cuprite mining dataset collected by the Airborne Visible Infrared Imaging Spectrometer (AVIRIS) (http://aviris.jpl.nasa.gov/html/aviris .freedata.html.) is used in the real data experiment to evaluate the unmixing performance of the proposed LRSSU algorithm. The part utilized in the experiment is a subset of the scene, with $100 \times 100$ pixels, including 224 bands in the range of $0.4-2.5 \mu \mathrm{m}$, with a spectral resolution of $10 \mathrm{~nm}$. The experiment excludes the low SNR and low water absorption bands; that is, the 1-2, 105-115, 150-170, and 223-224 bands are removed, leaving 188 spectral bands. The spectral library utilized in the experiment is selected from the USGS library, which contains 240 spectral signatures representing different minerals. Low SNR and low water absorption bands are removed from the library in the same way, and 188 bands are left. Since the true abundance maps of the Cuprite dataset are not available, we will refer to the mineral classification map (as shown in Figure 6) generated by Tricorder software (http://speclab.cr.usgs.gov/ PAPER/tetracorder.) to qualitatively analyze the performance of different unmixing algorithms [32]. The experiment meets the maximum number of iterations to ensure the convergence of each unmixing algorithm.

The regularization parameters involved in SUnSAL, CLSUnSAL, and DRSU algorithms are empirically set to $\lambda$ $=0.001, \lambda=0.01$, and $\lambda=0.0001$, and the parameters related to SUnSAL-TV, JSpBLRU, and LRSSU algorithms are empirically set to $\left(\lambda=0.001, \lambda_{\mathrm{TV}}=0.001\right)$ and $(\lambda=0.05$ $, \tau=0.02)$, and $(\lambda=0.08, \tau=0.02)$, respectively. Figure 7 takes three typical minerals: alunite, buddingtonite, and 


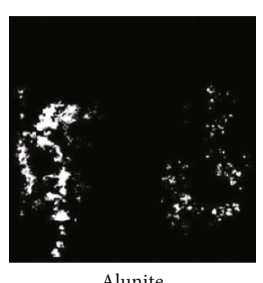

Alunite

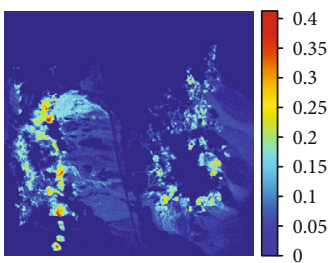

SUnSAL

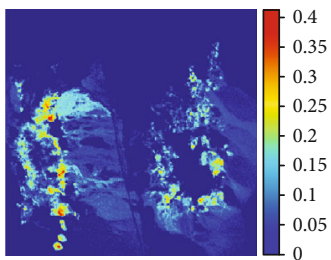

CLSUnSAL

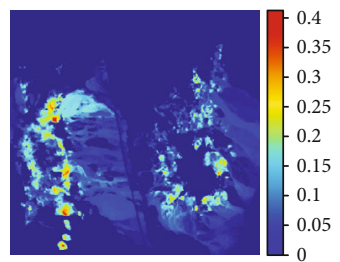

SUnSAL-TV
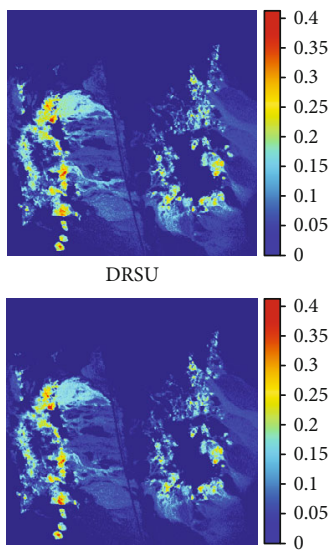

JSpBLRU

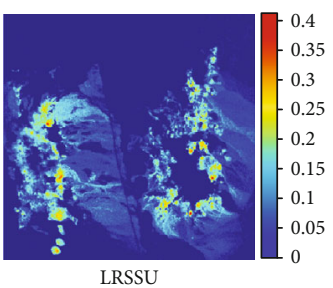

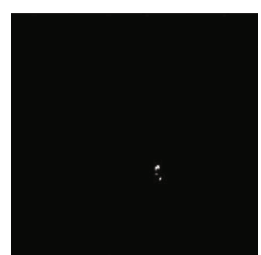

Buddingtonite

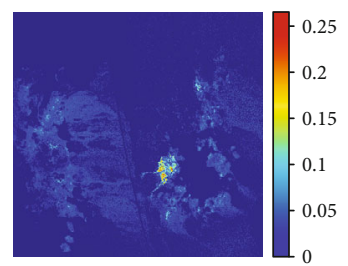

SUnSAL

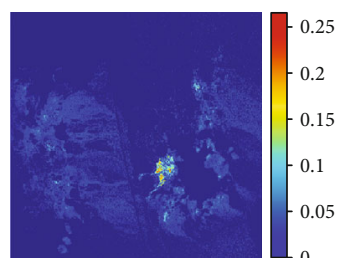

CLSUnSAL

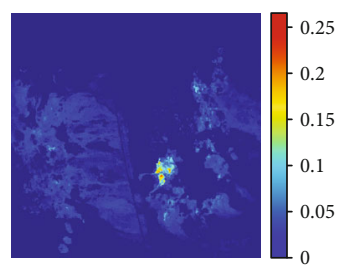

SUnSAL-TV

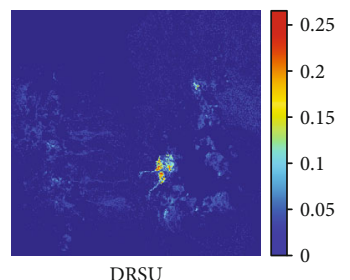

DRSU

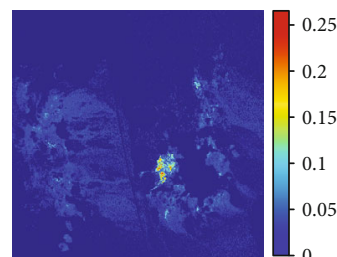

JSpBLRU

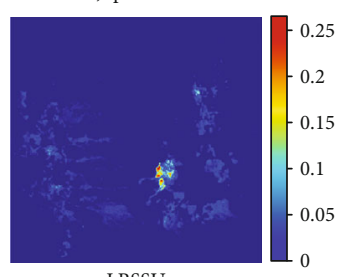

LRSSU

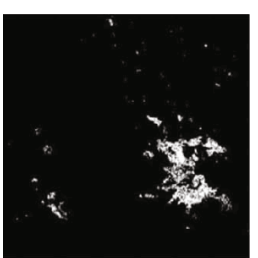

Chalcedony

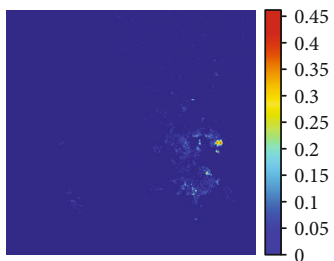

SUnSAL

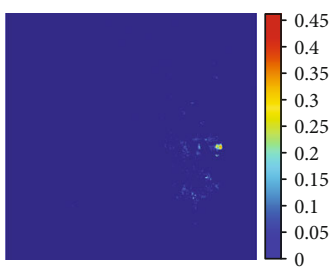

CLSUnSAL

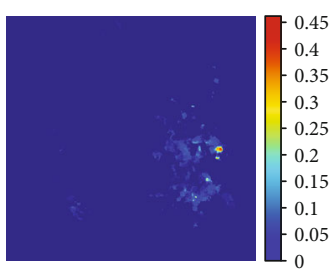

SUnSAL-TV

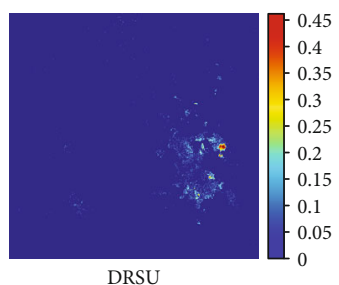

DRSU

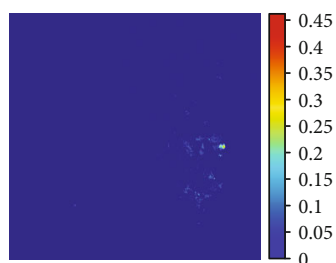

JSpBLRU

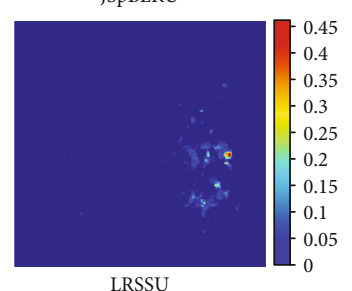

LRSSU

FIGURE 7: Fractional abundance maps estimated by different unmixing algorithms for the Cuprite subscene. 
chalcedony, as examples to show the abundance maps obtained by unmixing the Cuprite dataset with the above algorithms. From Figure 7, we can observe that the abundance maps obtained by the SUnSAL and CLSUnSAL algorithms are noisy, especially in buddingtonite. The abundance maps obtained by the SUnSAL-TV algorithm are obviously oversmooth, such as alunite and buddingtonite. The abundance maps obtained by the DRSU algorithm have poor spatial consistency, such as chalcedony. In addition, the abundance maps estimated by the LRSSU algorithm are less disturbed by noise than the JSPBLRU algorithm that also adopts the low-rank constraint, such as buddingtonite, and exhibit better spatial consistency, such as chalcedony. The abundance maps of the proposed LRSSU algorithm are closest to the Tricorder reference maps among all comparison algorithms. The qualitative analysis for the real data experiment confirms that the LRSSU algorithm is effective in unmixing real hyperspectral data and gets better unmixing results.

\section{Conclusions and Future Work}

In this paper, we propose a new sparse reduced-rank regression model for hyperspectral unmixing, which merges spatial and spectral information into the regularizer to enhance the interpretation of hyperspectral data. A local spatial weighting factor is introduced in the collaborative sparse unmixing model, which promotes the spatial smoothness of the image. Moreover, a low-rank constraint is imposed on the abundance estimation to enhance the row sparsity of the abundance matrix, which to some extent overcomes the influence of the high correlation of spectral signatures in the library. The proposed LRSSU model is solved by an ADMM-based the inner and outer loop scheme, the inner loop updates fractional abundances, and the outer loop calculates the spatial weights. The scheme accelerates the convergence of the algorithm. Simulated and real hyperspectral data experiments reveal that the proposed LRSSU algorithm enhances the identification of actual endmembers in the spectral library. Compared with SUnSAL, CLSUnSAL, SUnSAL-TV, DRSU, and JSPBLRU algorithms, the proposed LRSSU algorithm improves the accuracy of abundance estimation and shows the advantages of the spectral-spatial multiple constraint strategy. In the future, we will extend the constraint strategy to blind unmixing and further explore the spectral-spatial structure information of the hyperspectral data cube represented by a third-order tensor.

\section{Data Availability}

The United States Geological Survey (USGS) library used in simulated and real data experiments is available online at http://speclab.cr.usgs.gov/spectral.lib06. The Airborne Visible Infrared Imaging Spectrometer (AVIRIS) Cuprite dataset used in the real data experiment is available online at http:// aviris.jpl.nasa.gov/html/aviris.freedata.html.

\section{Conflicts of Interest}

The author declares that there are no conflicts of interest.

\section{Acknowledgments}

This work was supported in part by the National Natural Science Foundation of China under Grant 61865012, in part by Basic Science and Technology Research Project of National Key Laboratory of Science and Technology on Automatic Target Recognition under Grant WDZC2020 5500204 , in part by Key Research and Development Program of Jiangxi Province under Grant 20202BBGL73081 and Grant 20181ACG70022, and in part by the Science and Technology Project of Jiangxi Provincial Department of Education under Grants GJJ170992 and GJJ190956.

\section{References}

[1] J. M. Bioucas-Dias, A. Plaza, G. Camps-Valls, P. Scheunders, N. M. Nasrabadi, and J. Chanussot, "Hyperspectral remote sensing data analysis and future challenges," IEEE Geoscience and Remote Sensing Magazine, vol. 1, no. 2, pp. 6-36, 2013.

[2] L. Drumetz, M. A. Veganzones, R. Marrero et al., "Binary partition tree-based local spectral unmixing," Hyperspectral Image and Signal Processing: Evolution in Remote Sensing (WHISPERS), 2014.

[3] J. M. Bioucas-Dias, A. Plaza, N. Dobigeon et al., "Hyperspectral unmixing overview: geometrical, statistical, and sparse regression-based approaches," IEEE Journal of Selected Topics in Applied Earth Observations and Remote Sensing, vol. 5, no. 2, pp. 354-379, 2012.

[4] D. Landgrebe, "Hyperspectral image data analysis," IEEE Signal Processing Magazine, vol. 19, no. 1, pp. 17-28, 2002.

[5] M.-D. Iordache, J. M. Bioucas-Dias, and A. Plaza, "Sparse unmixing of hyperspectral data," IEEE Transactions on Geoscience and Remote Sensing, vol. 49, no. 6, pp. 2014-2039, 2011.

[6] C. Deng, S. Zhang, W. T. ShengqianWang, and Z. Wu, "Sparse hyperspectral unmixing based on smoothed 10 regularization," Infrared Physics and Technology, vol. 67, pp. 306-314, 2014.

[7] F. Li, S. Zhang, B. Liang, C. Deng, C. Xu, and S. Wang, "Hyperspectral sparse unmixing with spectral-spatial low-rank constraint," IEEE Journal of Selected Topics in Applied Earth Observations and Remote Sensing, vol. 14, pp. 6119-6130, 2021.

[8] L. Qi, J. Li, Y. Wang, Y. Huang, and X. Gao, "Spectral-spatialweighted multiview collaborative sparse unmixing for hyperspectral images," IEEE Transactions on Geoscience and Remote Sensing, pp. 1-14, 2020.

[9] M.-D. Iordache, J. M. Bioucas-Dias, and A. Plaza, "Collaborative sparse regression for hyperspectral unmixing," IEEE Transactions on Geoscience and Remote Sensing, vol. 52, no. 1, pp. 341-354, 2014.

[10] S. Zhang, J. Li, H. Li, C. Deng, and A. Plaza, "Spectral-spatial weighted sparse regression for hyperspectral image unmixing," IEEE Transactions on Geoscience and Remote Sensing, vol. 56, no. 6, pp. 3265-3276, 2018.

[11] H. Li, R. Feng, L. Wang, Y. Zhong, and L. Zhang, "Superpixelbased reweighted low-rank and total variation sparse unmixing for hyperspectral remote sensing imagery," IEEE Transactions on Geoscience and Remote Sensing, pp. 1-19, 2020. 
[12] J. Liu, J. Zhang, Y. Gao, C. Zhang, and Z. Li, "Enhancing spectral unmixing by local neighborhood weights," IEEE Journal of Selected Topics in Applied Earth Observations and Remote Sensing, vol. 5, no. 5, pp. 1545-1552, 2012.

[13] R. Wang, H.-C. Li, W. Liao, and A. Pizurica, "Double reweighted sparse regression for hyperspectral unmixing," in 2016 IEEE International Geoscience and Remote Sensing Symposium (IGARSS), pp. 6986-6989, Beijing, China, July 2016.

[14] G. Martin and A. Plaza, "Spatial-spectral preprocessing prior to endmember identification and unmixing of remotely sensed hyperspectral data," IEEE Journal of Selected Topics in Applied Earth Observations and Remote Sensing, vol. 5, no. 2, pp. 380395, 2012.

[15] J. Li, C. Wu, R. Song et al., "Deep hybrid 2-d-3-d CNN based on dual second-order attention with camera spectral sensitivity prior for spectral super-resolution," IEEE Transactions on Neural Networks and Learning Systems, 2021.

[16] O. Eches, N. Dobigeon, and J.-Y. Tourneret, "Enhancing hyperspectral image unmixing with spatial correlations," IEEE Transactions on Geoscience and Remote Sensing, vol. 49, no. 11, pp. 4239-4247, 2011.

[17] X. Wang, J.-S. Pan, and S.-C. Chu, “A parallel multi-verse optimizer for application in multilevel image segmentation," IEEE Access, vol. 8, pp. 32018-32030, 2020.

[18] S. Zhang, G. Zhang, F. Li et al., "Spectral-spatial hyperspectral unmixing using nonnegative matrix factorization," IEEE Transactions on Geoscience and Remote Sensing, pp. 1-13, 2021.

[19] M.-D. Iordache, J. M. Bioucas-Dias, and A. Plaza, "Total variation spatial regularization for sparse hyperspectral unmixing," IEEE Transactions on Geoscience and Remote Sensing, vol. 50, no. 11, pp. 4484-4502, 2012.

[20] W. He, H. Zhang, and L. Zhang, “Total variation regularized reweighted sparse nonnegative matrix factorization for hyperspectral unmixing," IEEE Transactions on Geoscience and Remote Sensing, vol. 55, no. 7, pp. 3909-3921, 2017.

[21] S. Zhang, J. Li, K. Liu, C. Deng, L. Liu, and A. Plaza, "Hyperspectral unmixing based on local collaborative sparse regression," IEEE Geoscience and Remote Sensing Letters, vol. 13, no. 5, pp. 631-635, 2016.

[22] Q. Qu, N. M. Nasrabadi, and T. D. Tran, “Abundance estimation for bilinear mixture models via joint sparse and low-rank representation," IEEE Transactions on Geoscience and Remote Sensing, vol. 52, no. 7, pp. 4404-4423, 2014.

[23] L. Sun, F. Wu, T. Zhan, W. Liu, J. Wang, and B. Jeon, "Weighted nonlocal low-rank tensor decomposition method for sparse unmixing of hyperspectral images," IEEE Journal of Selected Topics in Applied Earth Observations and Remote Sensing, vol. 13, pp. 1174-1188, 2020.

[24] P. V. Giampouras, K. E. Themelis, A. A. Rontogiannis, and K. D. Koutroumbas, "Simultaneously sparse and low-rank abundance matrix estimation for hyperspectral image unmixing," IEEE Transactions on Geoscience and Remote Sensing, vol. 54, no. 8, pp. 4775-4789, 2016.

[25] J. Huang, T. Huang, L. Deng, and X. Zhao, "Joint-sparseblocks and low-rank representation for hyperspectral unmixing," IEEE Transactions on Geoscience and Remote Sensing, vol. 57, no. 4, pp. 2419-2438, 2019.

[26] R. G. Baraniuk, V. Cevher, M. F. Duarte, and C. Hegde, "Model-based compressive sensing," IEEE Transactions on Information Theory, vol. 56, no. 4, pp. 1982-2001, 2010.
[27] E. J. Candes and T. Tao, "Near-optimal signal recovery from random projections: universal encoding strategies?," IEEE Transactions on Information Theory, vol. 52, no. 12, pp. 5406-5425, 2006.

[28] S. Gu, L. Zhang, W. Zuo, and X. Feng, "Weighted nuclear norm minimization with application to image denoising," in 2014 IEEE Conference on Computer Vision and Pattern Recognition, pp. 2862-2869, Columbus, OH, USA, June 2014.

[29] J. M. Bioucas-Dias and M. Figueiredo, "Alternating direction algorithms for constrained sparse regression: application to hyperspectral unmixing," in 2010 2nd Workshop on Hyperspectral Image and Signal Processing: Evolution in Remote Sensing, pp. 1-4, Reykjavik, Iceland, June 2010.

[30] S.-C. Chu, D. Zhi-Gang, Y.-J. Peng, and J.-S. Pan, "Fuzzy hierarchical surrogate assists probabilistic particle swarm optimization for expensive high dimensional problem," KnowledgeBased Systems, vol. 220, p. 106939, 2021.

[31] P.-C. Song, J.-S. Pan, and S.-C. Chu, "A parallel compact cuckoo search algorithm for three-dimensional path planning," Applied Soft Computing, vol. 94, p. 106443, 2020.

[32] R. Clark, G. Swayze, K. Livo et al., "Imaging spectroscopy: Earth and planetary remote sensing with the USGS Tetracorder and expert systems," Journal of Geophysical Research, vol. 108, no. E12, pp. 5131-5135, 2003. 